\title{
La recherche-intervention pour une transition vers le développement durable
}

Intervention-research: an approach for transition towards sustainable development

Pierre M. Stassart, Marc Mormont et Daniel Jamar

\section{(2) OpenEdition}

\section{Journals}

Édition électronique

URL : http://journals.openedition.org/economierurale/432

DOI : 10.4000/economierurale.432

ISSN : 2105-2581

\section{Éditeur}

Société Française d'Économie Rurale (SFER)

Édition imprimée

Date de publication : 1 juillet 2008

Pagination : 8-22

ISSN : 0013-0559

Référence électronique

Pierre M. Stassart, Marc Mormont et Daniel Jamar, « La recherche-intervention pour une transition vers le développement durable », Économie rurale [En ligne], 306 | Juillet-août 2008, mis en ligne le 01 juillet 2010, consulté le 01 mai 2019. URL : http://journals.openedition.org/economierurale/432 ; DOI : 10.4000/economierurale.432 


\title{
La recherche-intervention pour une transition vers le développement durable
}

\author{
Pierre M. STASSART • Marc MORMONT • Département Socio-Économie, Environnement et Développe- \\ ment, Université de Liège, Belgique
}

Daniel JAMAR • Section Système agraire, Centre Wallon de Recherche agronomique

\section{Introduction}

L a question pratique traitée dans cet article -est celle de la viabilité d'une filière de production de viande bovine bio. Organisée par un distributeur, elle associe une vingtaine d'éleveurs - la plupart étant de nouveaux entrants dans ce secteur-, une coopérative de collecte, dirigée par quatre d'entre eux, un transformateur et le distributeur. Cette filière est confrontée à divers problèmes interdépendants : les éleveurs restent dans un référentiel technique très intensif (le Blanc Bleu Belge) et difficilement conciliable avec les exigences de la norme bio. Le distributeur leur propose une reconversion technique mais cette proposition se heurte à de multiples difficultés qui sont aussi bien relationnelles (confiance mutuelle), techniques (quel système de production ?), organisationnelles (rôle du transformateur) et commerciales (pour quel consommateur?). Cette situation a été traitée sur le mode de la recherche-intervention. Le sujet de cette étude n'est autre que le comment de cette démarche qui associe sociologue, agronome et économiste dans la recherche d'une transition vers une filière plus durable.

La recherche-intervention ${ }^{1}$ s'inscrit, en $^{\prime}$ partie, dans la lignée de la recherche-action. Celle-ci a généralement développé des recherches avec et pour des acteurs, dont l'ambition était de leur fournir des instruments cognitifs pour leur propre émancipation (Bradbury, 2004). La rechercheintervention, telle que nous la pratiquons, s'en distingue cependant de deux manières : d'une part, elle s'adresse à des collectifs hétérogènes dont l'organisation même fait débat et, d'autre part, elle soulève des questions qui mêlent différents types de connaissances (sociologique, économique, agronomique) et de savoirs (éleveurs, distributeur). Cette approche met alors l'accent sur les transformations mutuelles de connaissances et de savoirs qu'elle génère entre disciplines et acteurs.

Mais l'enjeu est bien de produire une connaissance au service des acteurs, et donc ce qu'on va appeler une connaissance actionnable par eux. Dans un contexte hétérogène et conflictuel, des questions éthiques se posent. Elles ne seront évoquées qu'au passage. Mais ce n'est pas la seule justification d'une démarche d'intervention. Celle-ci se justifie aussi par la complexité de la situation. Par complexité, nous n'entendons pas seulement la multiplicité des variables et de leurs interactions, ce qui n'est pas original ; mais le fait qu'il s'agit là d'une action à laquelle la recherche participe, il n'existe, au départ, aucun point de vue à partir duquel on peut prétendre se donner une représentation stable de la situation et des possibilités d'évolution. La complexité est donc celle d'un acteur qui ne

1. La recherche a reçu l'appui financier de l'Agence nationale de la recherche (ANR) dans le « Programme agriculture et développement durable », projet ANR 05 PADD-0602 intitulé "Collectif de consommateurs et consommation durable ». Elle a fait suite aux travaux entamés dans le cadre d'un projet sur l'agriculture biologique qui s'inscrivait dans le " Programme d'appui au développement durable » du Service fédéral belge de la Politique scientifique. 
peut anticiper les réactions d'autres acteurs ni leurs conséquences. Et cette complexité renvoie notamment à la difficulté à définir les frontières du champ de l'intervention (Godard et Hubert, 2002). La rechercheintervention va donc se développer en cherchant à définir un système permettant à la fois de connaître et d'agir, c'est-à-dire mettre en œuvre une action collective.

Cette action collective comprend une production de connaissances à propos de ce qui la compose, qu'il s'agisse de règles ou de techniques : l'action modifie les connaissances, mais celles-ci réorientent l'action. La question centrale est de savoir comment conduire le processus, comment formuler les problèmes et comment capitaliser des connaissances (Aggeri, 2001).

Le dispositif de recherche est alors crucial à construire, à l'interface entre recherche et action. C'est cette construction simultanée de connaissances et d'une action que nous allons détailler. Notre propos est de définir un protocole de recherche bien qu'en réalité le processus dont il est question est plus progressif. Ce protocole est en fait une manière structurante de rendre compte d'une histoire continue faite de déplacement et de glissement.

Nous détaillons ce protocole ou dispositif de recherche en trois temps. Le premier temps comporte un diagnostic partiel, moment où les chercheurs vont identifier les recherches possibles : c'est le passage des questions dites «chaudes » aux questions « froides ». Le deuxième temps est celui de la construction d'un partenariat : les chercheurs proposent aux acteurs une démarche à la fois d'organisation et de recherche : c'est le passage à l'action collective. Le troisième temps est celui du bilan, des résultats auxquels aboutit cette démarche de recherche-intervention, en montrant comment ceux-ci portent avant tout sur la question de la transition vers la durabilité, via l'identification de points de bifurcation dans une trajectoire de développement.

\section{Construire un dispositif de recherche Processus de traduction et d'attachement}

Le dispositif de recherche n'est pas donné. Sa construction progressive suppose une série de traductions (Callon, 1986) qui impliquent des théorisations provisoires. Cette opération comporte deux étapes. Il s'agit d'abord d'identifier des questions qui peuvent faire sens pour les acteurs à partir de leurs points de vue différents : phase de diagnostic des préoccupations des uns et des autres. Ces questions doivent être ensuite transformées en étapes d'action et en propositions de recherche : étapes d'action pour les acteurs où les chercheurs jouent un rôle, et propositions de recherche auxquelles les acteurs doivent être intéressés pour qu'elles nourrissent les étapes suivantes.

\section{Traduire des préoccupations en questions situées}

Le dispositif s'agence à partir de la construction d'un espace de problèmes que les acteurs reconnaissent. Vu la nature a priori hétérogène et conflictuelle des relations, le chercheur doit justifier son intervention. Ensuite, il devra développer une compétence à collecter des données avec des acteurs dont les capacités d'organisation et de prise de parole sont très inégales. Enfin, la construction de cet espace suppose de définir un périmètre de diagnostic pertinent : la durabilité relève-t-elle ici d'une approche par la filière, par le territoire ou par le secteur? À ce stade, le choix opéré par les chercheurs doit reposer sur la mise à l'épreuve d'une théorisation progressive.

\section{Légitimer le diagnostic initial}

Les chercheurs s'appuient sur un programme de recherche du Service public fédéral de la recherche portant sur les modes de production et de consommation durables, lui-même adossé à un Plan politique de soutien au développement de l'agriculture biologique. Celle-ci, y compris au niveau européen, est 
définie comme contribuant au développement durable et elle reçoit à ce titre des primes spéciales. Ces options politiques légitiment la recherche. Mais elles ont aussi des effets puissants sur le secteur : en effet les primes à la reconversion ont incité de nouveaux producteurs à entrer dans ce secteur, et ceci rencontre la demande de la grande distribution. Mais ces « conversions » à l'agriculture biologique sont de natures diverses et la filière n'est pas stabilisée : les entrants sont nécessairement différents des agriculteurs bio « historiques » et la filière est en rupture par rapport à l'organisation très décentralisée et en circuit court des bios traditionnels. Les chercheurs prennent en compte aussi ces tensions internes au secteur, tensions qu'ils doivent lier à leur problématique. La première «traduction » va se faire « au nom du développement durable », label que ne peut refuser aucun des partenaires. Ce lien est un lien faible, mais il autorise cependant les chercheurs à établir des relations « exploratoires » avec les acteurs, relations suffisantes pour établir un premier diagnostic partiel.

Ce diagnostic initial consiste à interroger la diversité des points de vue des acteurs, qu'ils soient impliqués, soit en tant que parties prenantes dans l'élevage bio et ses filières de commercialisation (débats publics et interviews de chefs d'entreprises) soit affectés vers le secteur des consommateurs (Grin et al, 1997). Pour éviter une confrontation brutale, les chercheurs interrogent séparément différentes catégories d'acteurs qui sont en fait éloignés les uns des autres soit par des rapports de distance (producteurs-consommateurs), soit en raison de relations conflictuelles (éleveurs-distributeurs). Et, pour ne pas être enfermés dans le système existant, ils ne se limitent pas aux acteurs organisés et à leurs représentants. L'éthique de la parole à tous rejoint ici une ouverture scientifique à toutes les questions susceptibles de peser.

Les informations collectées sont surtout des questions, qui vont faire l'objet de mises en débat dans des instances intermédiaires constituées au sein du dispositif de recherche : un « comité de suivi » chargé de valider les choix opérés en cours de recherche, et un « groupe de compétence » constitué à l'initiative des chercheurs pour envisager la pertinence des interrogations retenues, compte tenu de leur connaissance du secteur. L'espace des problèmes devient ainsi un nouvel espace de relations et d'échanges. Cette phase conduit à plusieurs types de résultats que les chercheurs vont interpréter.

L'hétérogénéité des préoccupations impose une lecture sociologique. Il s'agit d'opérer un cadrage progressif qui rend compte des observations révèlant tensions et incohérences dans l'organisation (Stassart et Jamar, 2005). Là où les économistes parlent convention ou coûts de transaction pour analyser l'organisation d'une filière, nous mobiliserons comme cadre interprétatif le concept de référentiel (Jobert et Muller, 1989). Ce concept possède deux caractéristiques :

- il met l'accent sur la cohérence d'un secteur, cohérence faite du mixte des croyances, des savoirs et des normes partagés, mais aussi sur la capacité d'interprétation de chaque partenaire, ce qui ouvre la question de ce qui est non partagé (et potentiellement conflictuel) ;

- il relie sectoriel et global, donc le processus de traduction d'un impératif global dans un secteur spécifique.

L'entrée de nouveaux agriculteurs dans l'agriculture biologique à la fin des années 1990, suite à l'octroi des primes à la reconversion et au développement du marché bio, a fait passer les éleveurs d'un référentiel à l'autre et les oblige non seulement à une reconversion économique, mais aussi à une conversion à la fois technique et mentale. Ceci pour deux raisons.

- D'une part, des travaux antérieurs (Stassart, 2003) avaient montré la grande cohérence et la rigidité du système de production conventionnel en élevage bovin belge, du 
fait d'une instrumentation forte dans des techniques, des normes et, in fine, une définition de la qualité du produit à laquelle les consommateurs adhèrent massivement.

- D'autre part, la construction d'une filière bio de type industriel (division du travail entre éleveurs, transformateurs, distributeurs) entre en opposition avec les valeurs « classiques » du bio fondées sur le circuit court, le rapport en face à face entre producteurs et consommateurs.

Ces exigences contraires de conversion et d'équipement de la filière longue « bio » de type grande distribution amènent alors à centrer nos observations à l'intérieur d'un périmètre qui est celui de la filière. Celui-ci permettait, en effet, de saisir le contexte dans lequel des dynamiques de transformation engageaient les acteurs de l'élevage bio. Que produit ce diagnostic exploratoire portant sur la filière?

\section{Des préoccupations \\ aux modèles de recherche}

Les préoccupations collectées sont brutes, peu construites : elles sont l'expression d'insatisfactions, de difficultés, de souhaits, d'attentes. Chacune exprime un questionnement porté par un ou plusieurs acteurs parce qu'il fait sens à leurs yeux. On distingue d'abord des incohérences, c'està-dire des décalages entre les composantes du système ou de la filière. Des incohérences normatives se manifestent entre pratiques et règles (certaines normes bio ne sont pas respectées, par exemple l'obligation de pâturage) ainsi que des incohérences cognitives (la volonté de distinguer le produit bio en termes de «qualité » perçue par le consommateur, alors que le produit est très standardisé). On distingue ensuite des tensions, c'est-à-dire des désaccords qui opposent les acteurs, tels que le désaccord entre éleveurs et distributeurs sur la confiance mutuelle qu'ils se doivent, ou le désaccord entre producteurs et distributeurs sur la définition du consommateur et de ses attentes. Ces désaccords ne sont néanmoins pas uniquement le fait de points de vue différents s'exprimant sur une réalité commune. Ces différences renvoient aussi à des objets de niveaux de complexité différents. Ainsi, la demande du consommateur peut-être réduite à une question de définition de part de marché du bio, ou au contraire se complexifier en interrogeant sur ce qui construit les demandes des consommateurs. La distinction de cette « double distance » (Darré, 1997) renvoie alors à la nécessité de clarifier les niveaux d'opposition de points de vue. Bawden (1997) définit trois niveaux de complexité, que l'on peut illustrer ici en reprenant les préoccupations des acteurs portant sur la qualité du produit.

- Premier niveau de complexité. La préoccupation sur les critères de qualité existants : valider et communiquer sur l'absence de résidus dans le produit bio, abaisser sous le plafond réglementaire, le pourcentage non-bio dans l'aliment pour bétail d'élevage bio, renforcer les contrôles de certification. La question est " comment améliorer ce que l'on fait?"

- Second niveau de complexité. La préoccupation sur la cohérence de la coopération au sein de la filière : s'organiser autour d'un projet de qualification qui concerne soit la régularité du produit, soit sa différenciation, soit sa crédibilité. La question pratique posée est celle du « comment et avec qui faire d'une autre manière ce que l'on fait? ».

- Troisième niveau de complexité. L'interrogation sur les hypothèses sous-jacentes au modèle d'action : questionner plus fondamentalement le modèle d'action de la filière, les possibilités de collaboration souhaitables entre acteurs (constitués ou non), et plus spécifiquement entre éleveurs, supermarchés et transformateurs industriels. La question n'est plus celle du comment, mais du «pourquoi faire ce que l'on fait et pourquoi pas le faire d'une autre manière?». 
Ces trois niveaux de problématisation ne s'excluent pas, mais leur portée en termes d'apprentissage diffère parce qu'ils vont renvoyer à trois modèles différents de recherche (Hatchuel 2000). Le premier niveau de complexité vise une vérité objectivable, détachable des contingences. C'est le « Modèle I du laboratoire » (physique, chimie, agronomie) où le chercheur isole des entités qu'il peut manipuler. L'expérimentation ou l'analyse produit de la connaissance dont la légitimité dépend de son indépendance, et la valeur de son degré de généralité. Mais face à la complexité biotechnique et sociale d'un monde qu'il ne peut isoler et manipuler, le chercheur est amené à naturaliser son objet. Il développe des outils d'observation capables de rendre compte de la cohérence et de la dynamique de systèmes. La vérité, c'est la recherche de cohérence dans la complexité qui se substitue à la recherche de l'objectivité dans l'expérimentation. C'est le «Modèle II de terrain » (écologie, sciences sociales) qui répond au deuxième niveau de complexité. Cependant, la description d'un monde complexe dépend à son tour de l'état des connaissances et des modes d'organisation dont on dispose. La distinction entre description et prescription devient difficile. S'intéresser à la transformation de mondes complexes vers plus de durabilité implique de s'intéresser à la transformation des connaissances explicites et implicites sur ce monde. Or, les dimensions spatiale et temporelle des objets étudiés renvoient à l'incomplétude de ces connaissances et de nos états de référence. Il n'y a plus une vérité mais des vérités. C'est le « Modèle III de l'action collective » qui correspond au troisième niveau de complexité. Le chercheur, parce qu'il reconnaît la limite de ses connaissances et leur caractère évolutif, est partie prenante de la diversité des mondes qu'il décrit. Ce n'est plus le terrain qui est central, mais c'est la manière dont les acteurs s'associent et interagissent dans le temps et l'espace pour définir un problème en fonction de leurs connaissances. Il y a alors une méthodologie mais pas de solution préprogrammée.

L'art de la recherche-intervention est alors l'art de traduire les préoccupations des acteurs en questions de recherche, et de les traiter chacune dans l'un des trois modèles de recherche décrits. La rechercheintervention impose ensuite de construire sur certaines questions un point d'entrée à l'action collective et simultanément d'introduire dans celle-ci des résultats de recherches menées sur le modèle du laboratoire ou celui du terrain.

\section{2. Élaborer une proposition de recherche}

La mise en œuvre de la recherche-intervention est une action collective qui renvoie à deux conditions (Hatchuel, 2002) ${ }^{2}$ :

- elle suppose que les acteurs puissent s'engager sur une question ouverte pour l'ensemble d'entre eux : un des problèmes, porté par certains acteurs, doit pouvoir être transformé en un questionnement collectif, - de façon symétrique, ce questionnement doit pouvoir être relié à l'agenda des chercheurs, en l'occurrence aux dimensions de développement durable.

\section{Construction d'un cadre d'action}

Une question ouverte est une question pour laquelle les acteurs sont prêts à se poser une question de niveau de complexité III : «Pourquoi faire ce que l'on fait et pourquoi pas le faire d'une autre manière? ». Dite dans des mots simples, la question clef de cette recherche-intervention pourrait être le modèle d'action de la filière : le distributeur a-t-il comme partenaire privilégié l'industriel intermédiaire ou bien les éleveurs ? Sur cette question, l'action collective peut émerger parce que cette question fait l'objet de points de vue différents entre éleveurs et distributeur, mais elle est un enjeu pour tous : pour le supermarché c'est se rapprocher des éleveurs pour mieux qualifier et

2. $C f$. Page 38 . 
sécuriser son produit ; pour les éleveurs, c'est une question de confiance dans le distributeur et dans le transformateur, mais aussi de relation avec la coopérative de collecte gérée par quatre d'entre eux, interlocuteurs exclusifs du distributeur. Les tensions sont donc multiples, mais elles ne suffisent pas à mettre en mouvement le collectif. Pour que l'objet « relation éleveurs-distributeur » devienne actionnable, il faut construire une proposition d'action légitime.

La filière bio que nous suivons a un pilote, le distributeur. Les autres acteurs le considèrent comme tel. Pour les éleveurs, il est légitime parce qu'il peut parler au nom du marché et des consommateurs qu'il représente et qu'il a le pouvoir d'un commanditaire face au sous-traitant qu'est le transformateur industriel. Il a d'ailleurs imposé à ces derniers une grille de prix des bovins bio « sur pied » jugée très avantageuse par les éleveurs. Le distributeur a donc une force légitime de proposition. Ce n'est pas seulement une force d'imposition, mais plutôt une capacité et une compétence que Hatchuel qualifie de prescription faible. La prescription « faible » consiste à fixer non pas des buts, des missions ou des procédures mais des « objets de travail » qui sont aussi des «objets de connaissance » : elle permet une orientation générale et une répartition provisoire des tâches et des zones de compétences (Hatchuel, op . cit.). Cette prescription est double :

- passer d'un modèle d'organisation industrielle de la filière, qui dissocie les activités d'élevage (reproduction) de celles d'engraissement, à un nouveau modèle fait exclusivement de fermes qui associent naissage et engraissement ${ }^{3}$,

- convertir les troupeaux du Blanc Bleu Belge vers une race rustique bien différenciable sur le marché : la Limousine. Cette

3. Cette transformation intéresse le distributeur, parce qu'en réduisant le nombre d'intermédiaires, elle permet de sécuriser la filière. double proposition redéfinit les zones de compétences : elle privilégie un partenariat commercial direct avec un groupement d'éleveurs bio représentés par une coopérative de collecte plutôt qu'avec l'intermédiaire industriel conventionnel, qui devient alors son transformateur sous-traitant.

Mais cette prescription n'est, en l'état, pas actionnable. Mise à l'épreuve, elle se heurte aux divergences des univers cognitifs des acteurs et aux tensions que nous avons relevées. Pour être actionnable, cette proposition doit permettre d'exprimer chaque point de vue avec suffisamment d'efficience, à la fois pour chaque acteur-maillon de la filière et dans les échanges entre acteurs.

C'est ici qu'interviennent les chercheursacteurs en élaborant une transaction qui redéfinit la situation et le rapport coûts/bénéfices que ces acteurs peuvent en tirer. Le principe d'une transaction repose sur l'idée que l'on peut, en présence d'intérêts divergents, construire un consensus supposé en élaborant un objet commun qui implique des relations à la fois partiellement complémentaires et partiellement opposées (Rémy 1978). Ainsi la transaction autour des tensions de différenciation et de la relation éleveur-grande distribution s'appuie sur la prescription faible initiale du distributeur : changement d'organisation (naisseurengraisseur) et changement de race (limousine) mais elle va proposer de transformer les relations entre acteurs.

La prescription faible est utilisée par les différents acteurs à partir du point de vue que leur univers développe : différenciation du marché pour le distributeur, exclusivité pour la coopérative de collecte et orientation claire quant à la conversion de race (du Blanc-Bleu Belge au Limousin) liée à la garantie d'un débouché pour les éleveurs. Elle leur permet de développer et de mettre en valeur leur point de vue vis-à-vis des acteurs qui ont un point de vue différent, et ainsi d'affirmer leur identité. La transaction réorganise la proposition en la structurant au sein d'une action collective qui va 
impliquer de façon contractuelle quatre acteurs et leur identité. Cette transaction en quatre points rend la prescription faible adoptable.

- Le distributeur endosse explicitement la responsabilité du comportement du transformateur industriel, l'abattoir. Ceci instaure un statut de sous-traitant de l'industriel, dont les éleveurs bio se méfient en raison de ses pratiques opaques.

- La coopérative de collecte bio, gérée par une minorité d'éleveurs, accepte que la vingtaine d'éleveurs dont elle s'est toujours fait le seul porte-parole, deviennent un collectif à part entière, dissocié de la coopérative.

- Les éleveurs, bénéficiant d'une certaine autonomie par rapport à la coopérative de collecte, constituent alors un groupement, une entité nouvelle partie prenante de l'action collective.

- Les chercheurs, en échange de leur collaboration avec le distributeur, renoncent à faire interagir consommateurs et acteurs de la filière impliqués dans cette transaction. Ils se privent d'une interaction directe avec les consommateurs dans l'action collective, évitant ainsi la contradiction sousjacente entre les définitions implicites du consommateur chez les uns et les autres.

Ces quatre changements de relation au sein de la filière sont scellés dans une « convention de collaboration limitée » entre distributeur, coopérative de collecte, éleveurs bio et chercheurs. Si cette convention offre un cadre d'action collective, il reste alors à définir des objets actionnables qui s'inscrivent dans les préoccupations, pour les chercheurs, de durabilité.

\section{Action collective, équité \\ et durabilité environnementale}

Si la transaction réorganise le champ de la prescription initiale en modifiant les relations entre acteurs et en enrichissant la proposition de changement de race par celle de naisseurengraisseur, ce qui va la rendre « adoptable » (Teulier et Hubert, 2008), c'est la possibilité qu'elle offre, aux deux nouvelles entités, chercheurs et éleveurs, de la reprendre à leur compte. De plus, en l'adoptant, éleveurs et chercheurs vont dépolariser la situation initiale qui était caractérisée par une méfiance profonde entre distributeur et coopérative de collecte.

La proposition « changement de race » était en effet restée sans effet pendant cinq ans, parce que la prescription faible provenant du distributeur s'inscrivait dans une asymétrie trop forte entre un acteur en position oligopolistique (l'enseigne de grande distribution) et des acteurs atomisés (les agriculteurs). L'asymétrie bloquait la construction d'un processus de changement qui nécessitait d'avoir accès aux savoirs des différents acteurs (éleveurs, coopérative de collecte, transformateurs, distributeurs) pour les articuler. De plus, pris séparément, distributeurs, transformateurs et coopérative de collecte avaient en même temps de bonnes raisons de ne pas opter pour ce changement :

- incertitude sur la distribution des coûts et des bénéfices du changement pour la coopérative de collecte,

- contrainte initiale forte pour la réorganisation de la chaîne de transformation pour l'intermédiaire industriel,

- difficulté à organiser la régularité de la mise en marché pour le distributeur.

Réduire cette asymétrie exige de la part des chercheurs de mettre en place un dispositif capable de garantir une redistribution plus équitable des coûts-bénéfices du changement, et une articulation des savoirs hétérogènes à mobiliser. Cela revient à construire des engagements réciproques autour d'objets actionnables au sens d'Agyris (1996). L'équité dépend alors de la capacité du collectif à générer des objets d'action qui prennent en compte la diversité des savoirs et de leur légitimité. Comment avoir accès à ces savoirs ? La transaction opérée a fait émerger dans l'action collective l'association « éleveurs et chercheurs ». Les nouvelles relations qu'elle instaure - notamment des 
discussions entre éleveurs et distributeurs permettent alors aux chercheurs d'explorer, à travers les savoirs des différents acteurs, les objets de changements possibles. Cette possibilité ne suffit néanmoins pas. En effet, l'hétérogénéité des savoirs impose des compétences « dédiées à ces savoirs » c'està-dire des compétences ayant développé la capacité de capter ces savoirs. Cette capacité est définie pour partie par le choix de la démarche recherche-intervention que nous décrivons. Mais cette capacité ${ }^{4}$ à capter les savoirs hétérogènes et à les traduire suppose hétérogénéité et coordination des disciplines mobilisées. C'est alors le caractère interdisciplinaire du groupe de chercheurs composé d'un agronome, d'un sociologue et d'un économiste, et les interactions fortes et parfois tendues entre eux, qui forment cette capacité à capter la diversité des savoirs nécessaire pour générer ces objets actionnables dans le cadre des engagements réciproques (encadré 1).

Les chercheurs identifient, autour du changement de race, trois objets susceptibles d'induire des changements dans les relations :

- le bon d'accès (savoir d'éleveurs),

- le certificat d'inventaire de troupeau (savoir de la coopérative de collecte),

- la triple mention sur l'étiquetage produit (savoir de distributeur).

Ces trois objets matériels sont des objets sociotechniques qui incorporent les savoirs hétérogènes des acteurs en cours d'action. C'est leur caractère d'objets intermédiaires (Vinck, 1999) qui permet de construire des passages entre points de vue différents. Leur mise en œuvre met à l'épreuve les positions des acteurs, libère certains de leurs savoirs et les rend davantage interdépendants. Ils relient éleveurs, coopérative de collecte et distri-

4. L'interdisciplinarité dépasse la question du croisement intellectuel des disciplines : il s'agit de posture de recherche, de rapport aux acteurs, de circulation des questions et d'articulation des savoirs, aspects que nous ne pouvons développer dans le cadre de ce travail. buteur dans un système d'engagements réciproques qui garantissent une redistribution plus équitable des coûts-bénéfices.

L'équité est donc une condition à l'engagement d'acteurs qui sont toujours dans des rapports asymétriques par rapport aux enjeux de marché. Ce que l'on nomme communément «blocage » par rapport à des propositions de changement est souvent le signe d'une distribution trop inéquitable des coûts et bénéfices du changement, ce qu'illustre parfaitement l'exemple du refus des OGM alimentaires de première génération chez les consommateurs. Mais si changement il peut y avoir, quelle direction celui-ci veut-il prendre ? S'agit-il de développer une innovation ou d'améliorer la position compétitive des éleveurs bio belges ? S'agit-il de développer un marché de qualité différenciée ? La proposition changement de race, si elle est clairement connectée à des enjeux de marché pour les acteurs de la filière (stratégie de différenciation du distributeur), est simultanément connectée à des enjeux environnementaux pour les chercheurs.

Le changement de race est plus qu'un banal changement d'objet. Il permet de changer les pas de temps et d'espace, qui ont d'importantes conséquences sur la gestion des pâturages et de la biodiversité. Ainsi, la question de l'environnement, peu présente dans les enjeux de filière, peut être rouverte. Passer à la race limousine, c'est en effet changer de génétique et donc de capacité à valoriser un aliment : si le Blanc Bleu Belge est le plus performant pour valoriser l'aliment concentré, le limousin l'est davantage pour valoriser les prairies et donc l'entretien d'espaces ouverts qui font les paysages. De plus, quitter le Blanc Bleu Belge pour la race limousine, c'est accroître la durée de vie moyenne des vaches en pâture de 3 à 8 ans.

Ceci permet alors aux agronomes d'introduire une notion peu traitée en zootechnie, celle de la carrière d'un animal et du troupeau (Coulon et al, 1989). 


\section{Encadré 1. Savoirs - Objets intermédiaires - Réciprocité des engagements}

1. L'agronome, dans son travail de suivi en ferme, identifie avec les éleveurs une reformulation de la question du développement durable de la filière à la lumière de leur préoccupation d'équité. Se faisant leur porte-parole, il suggère de mettre en place un système de «bons d'accès » qui fonctionnerait selon le principe du quota de production. Ceci permettrait de garantir à ceux qui font le choix du changement de race un débouché dans la filière. Les bons d'accès sont un savoir « organisationnel » que certains éleveurs ont appris et expérimenté dans une autre filière « label fermier $\gg$.

- Les bons d'accès engagent la coopérative de collecte (et secondairement le distributeur) à donner une priorité aux éleveurs qui adoptent le changement ; c'est donc un facteur de redistribution des bénéfices du changement au profit des éleveurs qui se convertissent au limousin.

2. L'agronome et le sociologue identifient dans leur travail exploratoire avec la coopérative de collecte un second élément qui permet de valider l'engagement des éleveurs et du distributeur sur le critère « éleveurs-engraisseurs bio ». Les éleveurs bio impliqués dans la filière sont devenus « éleveurs-engraisseurs » bio. La coopérative de collecte mobilise son savoir administratif et organisationnel en suggérant une façon à la fois crédible et efficace de valider l'identité de « naisseur-engraisseur ». Elle propose que chaque éleveur s'engage à fournir un formulaire particulier capable de garantir le caractère « éleveur-engraisseur» de la production. Ce formulaire, dit « inventaire de troupeau », est délivré par l'Agence fédérale de la sécurité de la chaîne alimentaire sur simple demande de l'éleveur. Le statut public et administratif de ce document gratuit le rend crédible, dans un secteur qui ne brille pas toujours par sa transparence. Cette proposition modifie les relations au sein de la filière.

- Elle engage les éleveurs sur la fourniture du document (et secondairement le distributeur) sur l'exclusivité naisseurs-engraisseurs. C'est une redistribution des bénéfices du changement au profit des éleveurs-engraisseurs, et secondairement de la coopérative de collecte qui cherche cette exclusivité.

3. Le sociologue et l'économiste à travers les focus groupes consommateurs et leur étude sur la stratégie bio des enseignes de grande distribution (Stassart et Jamar, en cours; Aertsen et Van Huylenbroeock, 2007), proposent au distributeur de s'engager sur un troisième élément : créer une triple mention « race : ...»«éleveur : ...»- «engraisseur : ... » qui sera signalée systématiquement par étiquetage sur l'emballage du produit et communiquée par les autres canaux de communication du distributeur : campagne de promotion et bulletin d'information consommateur.

- Cette mention, par la promesse qu'elle fait au consommateur, engage le distributeur vis-à-vis des éleveurs et de la coopérative de collecte. Elle redistribue les bénéfices d'une meilleure différenciation/identification du produit sur l'ensemble de la filière et, en particulier, vers les éleveurs qui sont ceux qui seront mentionnés via la race et leur caractère éleveur et engraisseur. 
Sans entrer dans les détails techniques, on peut montrer que la notion de carrière permet d'ouvrir et d'étudier des questions liées à la gestion de la biodiversité et se situant précisément à la frontière entre les disciplines de zootechnie, de nutrition, d'écologie et de médecine vétérinaire. En effet, rallonger et étudier la carrière de l'animal offre de nouvelles perspectives en terme d'apprentissage, car brouter et surtout apprendre à brouter des pâturages plus bruts et plus diversifiés n'est pas inné (Meuret et al, 2007), cela s'apprend tout comme la vie en troupeau et le respect de sa hiérarchie. Les apprentissages concernent aussi la santé du troupeau en pâture. Ainsi l'acquisition d'une immunité par le troupeau face aux parasites et donc la limitation de l'usage de médications préventives agressives et rémanentes peut avoir un impact environnemental. En effet, d'importants résidus de ces médicaments insecticides (préventifs) se retrouvent dans les bouses des animaux traités (vaches) et peuvent avoir alors un impact très négatif sur la faune coprophage des prairies et la décomposition ultérieure de ces bouses (Guilbot, 1999). Finalement, le changement de race permet donc l'ouverture d'une double question, fermée au niveau des acteurs de la filière et qui pour cette raison était diagnostiquée comme une série d'incohérences (par rapport au cahier de charges bio) plutôt que révélatrice d'une tension (traduisant l'expression de différents points de vue). Ces incohérences concernent l'obligation de pâturage, la question relevant de la santé animale et des méthodes alternatives de soin aux animaux.

Ces questions, tout comme celle de la demande des consommateurs exclue par le distributeur, mais identifiées par les chercheurs comme étant importantes dans la perspective du développement durable, ne peuvent être traitées collectivement car elles ne sont pas prioritaires aux yeux des acteurs. Elles sont alors traitées de façon dissociée dans le modèle de terrain (encadré 2). Dans ce cadre, c'est la manière de problématiser qui définit ce que le chercheur va prendre en compte et qui précise les frontières de son terrain d'observation. Deux recherches ${ }^{5}-$ qui ne peuvent qu'être résumées - sont alors entreprises.

La recherche-intervention comme méthode d'approche de la question du développement durable, implique de la part des chercheurs d'assumer une double responsabilité sociale qui réponde aux deux conditions fixées par Hatchuel (2002). D'une part, ils ont construit un objet de recherche actionnable pour les acteurs : partant de leurs préoccupations, les chercheurs ont opéré une série de traductions (identification des tensions et incohérences, niveaux de complexité des problèmes identification de la prescription faible transaction et construction d'un cadre d'action) qui débouche sur une recherche dans le modèle de l'action collective. D'autre part, le choix de ces traductions est orienté par leur capacité à connecter leur préoccupation en matière de développement durable. Ces connections sont soit parfois réalisées directement, parce qu'en tant que parties prenantes de l'action collective, ils peuvent y traduire leurs questions comme n'importe quel autre acteur; soit parce qu'ils vont chercher à nourrir cette action collective par des recherches dissociées opérées selon le modèle de terrain, voire de laboratoire. Il nous reste maintenant à examiner dans la troisième et dernière partie à quel type de résultats mène ce dispositif de recherche.

5. En termes de production scientifique, les apports sont variables selon les disciplines. Nous renvoyons aux publications en cours (Jamar et al, 2006 ; Jamar et al, 2007a ; Jamar et al, 2007b ; Stassart et Jamar [en cours]). 


\section{Encadré 2. Recherche selon le modèle de terrain}

\section{Dispositif d'observation du système parasitisme}

Constatant une incohérence entre normes (cahier de charges bio) et pratiques d'éleveurs bio sur la gestion du parasitisme, les chercheurs considèrent que des modèles de gestion de la santé très différents sont en jeu et font mener une recherche de type modèle de terrain. Problématiser la question du parasitisme pour le chercheur revient à identifier de nouvelles interactions et à tenter de la caractériser par un processus d'intéressement.

1. Partant de l'intérêt des éleveurs pour les pesées en ferme, qui permettent de mesurer les performances des taureaux en phase d'engraissement, les agronomes proposent à ces derniers d'utiliser la pesée, non seulement pour mesurer les performances de gain de poids, mais aussi pour évaluer leur état de santé : la pesée est couplée à des prélèvements sanguins et coprologiques pour mesurer la présence des parasites et le taux d'infestation.

2. En échange de ce service, les éleveurs acceptent de faire peser en prairie les jeunes vaches destinées à la reproduction. Passant de l'échelle du lot d'engraissement (taureaux) à l'échelle du troupeau (vaches) au pâturage, les agronomes transforment les pas de temps et les entités observées. Passant du taureau à la vache de reproduction, et de l'individu «à l'étable » au troupeau « en pâture », les notions de carrière de l'animal et de troupeau sont introduites.

3. Ces mesures, connectées à des observations en prairies, sont mises en discussion entre éleveurs réunis deux fois par an en présence d'un vétérinaire homéopathe qui analyse principalement la relation entre vétérinaire, éleveurs et bovins pour ce qui concerne la santé des troupeaux.

Partant d'un suivi classique de pesée en ferme, les agronomes ont donc construit un espace d'observations qui transforme les relations entre humains (éleveur/technicien/vétérinaire) et non-humains (parasites/bovins/pâturages) pour avoir accès aux informations qui leur permettront de caractériser leurs interactions (Jamar et al, 2007a).

\section{Identification d'une figure de consommateur-négociateur}

La réalisation d'une série de «focus groups » permet au sociologue de formuler l'hypothèse selon laquelle les consommateurs potentiels de viande bovine bio en grande surface font leur arbitrage en fonction d'autres points de repère que celui de la seule certification. Ces consommateurs sont négociateurs plutôt que sélectionneurs. Parmi les repères pertinents pour eux, la race est en fait un puissant signal de différenciation. En revanche, le lien fait entre mention «éleveur » et mention « engraisseur » est un signal trop faible pour les consommateurs, mais il est néanmoins cohérent avec la stratégie de différenciation de l'enseigne mise en avant par les économistes. Ce lien « éleveur-engraisseur » est un principe organisateur qui engage le distributeur dans l'exclusivité qu'il accorde aux éleveurs bio (Stassart, 2007). 


\section{Discussion et conclusion : transition et bifurcation}

L'incertitude était le point de départ de cette recherche. Pour chacun des acteurs, l'incertitude principale était de savoir ce que pouvaient accepter les autres et où mènerait alors tel ou tel changement. Plutôt que de s'appuyer sur une vision normative du développement durable, qui jugerait à partir de critères et d'indicateurs stabilisés, la recherche vise plutôt à assurer et accompagner une transition vers une plus grande durabilité. Le défi est alors de rendre possible une action collective qui va porter sur les normes et les formes d'organisation, mais aussi sur les connaissances et les possibilités techniques.

Ainsi la mise en place de bons d'accès et d'autres outils organisationnels peut permettre d'établir une relation plus confiante et de donner aux éleveurs une maîtrise suffisante de leur organisation productive. C'est alors que le changement de race devient un pari acceptable où distributeur comme coopérative de collecte doivent rendre des comptes aux éleveurs, ceux-ci s'engageant à leur tour et de manière vérifiable à se conformer à leur nouveau statut de naisseur-engraisseur.

Le changement de race a été rendu possible en faisant converger sur ce processus une série de changements partiels et relativement indépendants : la confiance n'est pas une attitude psychologique, elle repose sur des objets intermédiaires des relations. L'exigence d'exclusivité que réclament les éleveurs est assurée par un contrôle des entrées et des sorties de l'abattoir. Cela sera élaboré par la mise en place d'une base de données qui s'appuie sur les informations fournies par les différents acteurs. En réaction, la constitution de cette base de données crée une forte interdépendance entre eux. La filière devient représentable en tant que telle. La base de données peut aussi servir d'instrument de planification de la production, permettant un ajustement anticipé aux fluctuations de la demande.
La filière ainsi confortée peut affronter des questions plus larges. Dans l'état antérieur, la flexibilité du marché était obtenue par un ajustement de l'alimentation du bétail en phase finale d'engraissement. Les éleveurs pouvaient accélérer cette phase en rendant leurs animaux disponibles à l'abattage dès 20 mois au lieu des 24 mois exigés, la marge de manœuvre étant acquise grâce à une alimentation précoce et intensive à base de concentrés. Or ceci est en contradiction avec le cahier de charges bio et son obligation de pâturage.

Cette pratique ne peut être étudiée qu'en réintroduisant deux variables largement laissées de côté par la recherche, jusque-là basée sur les préoccupations immédiates des acteurs. Des recherches parallèles sur la demande des consommateurs ont en effet fait apparaître que les consommateurs sont très sensibles à la donnée relative à l'alimentation des animaux. À leurs yeux, ce qui fait différence s'exprime en termes d'élevage « forcé » par opposition à une alimentation naturelle (prairie). Il s'agit alors, pour les agronomes et les zootechniciens, de développer d'autres recherches, sur un modèle expérimental (de type terrain), pour prouver qu'il est possible de mettre en œuvre un autre schéma d'alimentation qui permette d'atteindre une même flexibilité par une autre combinaison de pâturage et de concentrés. Finalement les résultats pour les acteurs peuvent être synthétisés sous forme de trois apports principaux.

- D'une part, la filière s'est stabilisée en ce sens que les relations entre les partenaires sont devenues moins conflictuelles, qu'une confiance les lie désormais dans un régime d'engagements réciproques (et pas seulement de négociations) à l'égard du développement de cette filière qui est devenue progressivement un bien collectif. Cette coopération accrue passe par des objets socio-techniques qui assurent ces engagements sur la base de savoirs que la filière reconnaît à chaque partenaire. 
- D'autre part, la filière s'inscrit désormais dans une perspective renouvelée. C'est une bifurcation qui s'est opérée avec le changement de race pour produire un référentiel nouveau, hybridant de manière plus stable le bio et l'organisant en filière longue. Cela satisfait différentes exigences des partenaires, spécialement en termes de différenciation du produit. Cette bifurcation a été rendue possible par la mobilisation des savoirs des différents acteurs.

- Enfin, c'est cette bifurcation qui permet aux chercheurs de réintroduire les questions qui pouvaient dès l'origine et de leur point de vue donner lieu à des investigations : à savoir la question de la durabilité de ce mode de production.

La recherche-intervention est une démarche dans laquelle les chercheurs portent leur propre ambition, ici de transition vers le développement durable. Les disciplines différentes ont aussi chacune leurs domaines d'intervention, leurs objets et leurs méthodes. Ce que fait l'intervention, c'est agir sur les relations entre les acteurs de manière à pouvoir traiter des changements à opérer à différents niveaux de complexité. Ces changements sont autant de questions qu'il faut adresser à différents collectifs de recherche qui associent chacun à leur manière les acteurs. La rechercheintervention est donc une dynamique dans laquelle tous les acteurs, y compris chercheurs, sont amenés à se présenter les uns aux autres au plus près de ce qui les fait agir. C'est une condition indispensable à un développement durable, c'est-à-dire un développement qui préserve la capacité d'action des collectifs ou encore ce que Thompson (1997) appelle l'intégrité fonctionnelle.

\section{Remerciements}

Les auteurs remercient Didier Stilmant, directeur de la Section Système Agraire, du Centre Wallon de Recherche agronomique. Sa perplexité a grandement contribué aux résultats présentés dans cet article. Ils remercient également, le Lentic (ULg) et le programme ADD (ANR) qui ont permis d'exposer leurs arguments lors de leur colloque "Intervenir dans le monde du travail : la Responsabilité Sociale d'un centre de recherche en sciences humaines ", Liège, 30 novembre 2006 et « La prise en compte des Acteurs et de leurs représentations dans le Developpement Durable », Montpellier, 26-27 juin 2007. 


\section{RÉFÉRENCES BIBLIOGRAPHIQUES}

Aertsens J., Van Huylenbroeock G. (2007). Comparison of marketing strategies of retailers of organic beef. In P. Stassart, M. Mormont, D. Jamar, D. Stilmant (Eds), « How can organic farming contribute to sustainable production and consumption patterns », Brussel, Belgian Science Policy, p. 45-54.

Aggeri F. (2001). Développement durable et gouvernement de l'environnement : la formation d'un nouvel espace d'action collective. Séminaire Condor, Paris.

Agyris C. (1996). Actionable knowledge: design causality in the service of consequential theory. Journal of Applied Behavior Science, vol. 32, $\mathrm{n}^{\circ}$ 4, p. 390-408.

Bawden R. « Learning to persist: a systemic view of development». In Stowell F., Ison R., Armson J., Holloway J., Jackson S. et McRobb S. (Eds), Systems for Sustainability, Plenum Press, New York and London, 1997, p. 1-5.

Bradbury H. (2004). Doing work that matters despite the obstacles: an interview with Riane Eisler. Action Research, vol. 2, n 2, p. 209-227.

Callon M. (1986). Éléments pour une sociologie de la traduction. La domestication des coquilles Saint-Jacques et des marins-pêcheurs dans la baie de SaintBrieuc. L'Année sociologique, vol. 36, p. 169-208.

Coulon J.-B., Landais E., Garel J.-P. (1989). Alimentation, pathologie, reproduction et productivité de la vache laitière : interrelations à l'échelle de la lactation et de la carrière. INRA, Production Animale, 2(3), p. 171-188.

Darré J.-P. (1997). Une condition de la Recherche-Action : la coopération sur la problématique et son évolution. In Albaladejo C. et Casabianca F. (Eds.), «Étude Recherche Systèmes. Agraires Développement $», \mathrm{n}^{\circ} 30$, prospective-participative, p. 177-182.
Godard O., Hubert B. (2002). Le développement durable et la Recherche Scientifique à l'INRA. Paris, Inra.

Grin J., Van De Graaf H., Hoppe R. (1997). Tecnology Assesment through Interaction: a Guide. Pays-Bas, Ratheneau Instituut.

Guilbot R. (1999). Les insectes des prairies : un maillon essentiel de l'écosystème prairial. Fourrages, n 160 , p. 403-416.

Jamar D., Decruyvenaere V., Stassart P., Stilmant D. (2006). Reconversion to organic farming, between organic rules and agro-food chain referential: how to fit out the organic fattening referencial? In Andreasen C., Elsgaard B., Søndergaard L., Hansen G. (Eds.) «European Joint Organic Congress », Odense 30-31 mai, Organic eprint, www.orgprints.org.

Jamar D., Stassart P., Decruyvenaere V., Stilmant D. (2007a). Transformation of gastro-intestinal parasites managment scheme in the context of suckling herd conversion to organic farming. In Stassart P., Mormont M., Jamar D., Stilmant D. (Eds), « How can organic farming contribute to sustainable production and consumption patterns », Brussel, Belgian Science Policy, 2007, p. 117-135.

Jamar D., Stilmant D., Baret Ph. (2007b). Fodder productions in organic suckling beef farming systems: impact of the motivation of farmers and extensions services on the conversion to organic production. EGF, paper presented at the General Meeting of the European Grassland Federation. Gent, 3-5 september 2007. Jobert B., Muller P. (1989). L'État en action. Paris, PUF.

Hatchuel A. (2000). Research, Intervention and the production of knowledge. In LEARN Group (Eds), « Cow Up a Tree: Knowing and Learning for change in Agriculture », Paris, Inra Edition, coll. Science Update, p. 55-68. 
Hatchuel A. (2001). Quel horizon pour les sciences de gestion? Vers une théorie de l'action collective. In David A., Hatchuel A., Laufer R. (Eds.), « Les nouvelles fondations des sciences de gestion », Paris, Vuibert, FNEGE, p. 7-44.

Hatchuel A., Le Masson P., Weil B. (2002). From knowledge management to designoriented organisations. International Social Science Journal, n 171, p. 25-37. Meuret M., Débit S., Agreil C., Osty P.-L. (2007). Éduquer ses veaux et génisses : un savoir empiriques pertinent pour l'agroenvironnement en montagne ? Natures, Sciences et Sociétés, vol. 14, p. 343-352.

Reason P., Bradbury H. (Eds.) (2001). Handbook of action research. London, Sage.

Rémy J., Voye L., Servais E. (1978). Produire ou reproduire, Sociologie de la vie quotidienne. Bruxelles, EVO.

Stassart P. (2003). Produits fermiers : entre qualification et identité. Bruxelles, Peter Lang, coll. Ecopolis.

Stassart P. (2007). Transforming the figure of the consumer to reconfigure a chain. In Stassart P., Mormont M., Jamar D. et Stilmant D. (Eds), « How can organic farming contribute to sustainable production and consumption patterns », Brus- sels, Belgian Science Policy, p. 79-96. Stassart P., Jamar D. (2005). Equiper des filières durables? L'élevage Bio en Belgique. Natures Sciences et Sociétés, vol. 13, n 4, p. 413-420.

Stassart P., Jamar D. (en cours). Dynamiques conventionnelles : missing protocoles et systèmes de légitimité, relation éleveurs bio grande distribution.

Stassart P., Mormont M., Jamar D., Stilmant D. (2007). How can organic farming contribute to sustainable production and consumption patterns. Brussels, Belgian Science Policy.

Teulier R., Hubert B. (2008). Des concepts intermédiaires pour la conception collective. In Mélard F. (Eds.) « Écologisation, Objets et concepts intermédiaires », Bruxelles, Editions Peter Lang, coll. Ecopolis. p.163-186

Thompson P.-B. (1997). The Varieties of Sustainability in Livestock Farming. In «Livestock Farming Systems; More than Food Production », $4^{\text {th }}$ International Livestock Farming Systems Symposium, Foulum (Denmark), 22-23 août 1996, EAAP, Wageningen, 1997.

Vinck D. (1999). Les objets intermédiaires dans les réseaux de coopération scientifique. Revue française de sociologie, vol. XL, n 2, p. 385-414. 